\title{
Metastable strings and dumbbells in supersymmetric hybrid inflation
}

\author{
Wilfried Buchmüller \\ Deutsches Elektronen-Synchrotron DESY, \\ 22607 Hamburg, Germany \\ E-mail: buchmuwi@mail.desy.de
}

ABSTRACT: We study symmetry breaking and topological defects in a supersymmetric model with gauge group $\mathrm{U}(2)$, which can be identified with the right-handed part $\mathrm{SU}(2)_{R} \times$ $\mathrm{U}(1)_{B-L}$ of an extended electroweak symmetry of the Standard Model. The model has two phases of hybrid inflation terminated by tachyonic preheating where either monopoles and strings or, alternatively, dumbbells are formed. In the first case a stochastic gravitational wave background is predicted in the LIGO-Virgo band, possibly extending to the LISA frequency band and to nanohertz frequencies, which is generated by a metastable cosmic string network. In the second case no topological defects survive inflation and no stochastic gravitational wave background is produced.

KeYwords: Cosmology of Theories beyond the SM, Supersymmetric Gauge Theory

ArXiv EPrint: 2102.08923 


\section{Contents}

1 Introduction 1

2 Symmetry breaking in a supersymmetric $U(2)$ model $\quad 3$

3 Hybrid inflation and the formation of monopoles, strings and dumbbells $\begin{array}{lr}\text { in tachyonic preheating } & 8\end{array}$

3.1 Formation of metastable strings 9

$\begin{array}{lll}3.2 & \text { Formation of dumbbells } & 11\end{array}$

4 Conclusions 13

\section{Introduction}

The formation of topological defects is a generic feature of cosmological phase transitions [1]. While monopoles and domain walls would overclose the universe and must therefore be avoided, cosmic strings evolve towards a scaling regime where their fraction of the total energy density is constant. Cosmic strings have characteristic signatures in gravitational lensing, the cosmic microwave background and the stochastic gravitational wave background (SGWB) and are therefore a potentially very interesting messenger from the early universe (for reviews and references, see, for example, [2, 3]).

Symmetry breaking in grand unified theories (GUTs) can produce many kinds of topological or non-topological defects, including Nielsen-Olesen strings [4], 't Hooft-Polyakov monopoles [5, 6], unstable "dumbbells" or "X-strings" connecting a monopole-antimonopole pair [7], or "necklaces" where more than one string is attached to a monopole [8]. The monopole problem can be solved by inflation which is naturally connected to the GUT scale in supersymmetric hybrid inflation $[9,10]$. Moreover, it has been shown for a large class of GUTs that in spontaneous symmetry breaking schemes solving the monopole problem cosmic string formation is unavoidable [11].

The seesaw mechanism [12-14] connects the GUT scale with neutrino masses, and the related leptogenesis scenario [15] provides an elegant explanation of the cosmological baryon asymmetry (for a recent review, see [16]). The masses of the heavy seesaw partners of the light neutrinos depend on the breaking scale of $B-L$, the difference of baryon and lepton number. In a detailed study $[17,18]$ it was shown that cosmological $B-L$ breaking, starting from a false vacuum of unbroken $B-L$, can lead to a consistent picture of hybrid inflation, leptogenesis and dark matter. The spontaneous breaking of $\mathrm{U}(1)_{B-L}$ is tied to the formation of a cosmic string network and the corresponding SGWB was evaluated in [19].

Recently, it was pointed out that the seesaw mechanism and leptogenesis can be tested by means of metastable cosmic strings [20] that evade the tension between GUT-scale 
strings and the upper bound on the string tension from pulsar timing array (PTA) experiments [21-23]. Embedding the $\mathrm{U}(1)_{B-L}$ model into an $\mathrm{SO}(10)$ GUT, the $B-L$ strings become metastable [24]. They decay by quantum tunneling into string segments connecting monopole-antimonopole pairs. In the semiclassical approximation the decay rate per string unit length is given by [25-28]

$$
\Gamma_{d}=\frac{\mu}{2 \pi} \exp (-\pi \kappa), \quad \kappa=\frac{m_{M}^{2}}{\mu},
$$

where $m_{M}$ is the monopole mass and $\mu$ is the string tension. Using this decay rate and the BOS model [29] for GW emission from string loops (for a general discussion, see [3]), the SGWB generated by a metastable string network was evaluated for the parameters of the $B-L$ model. In the LIGO-Virgo frequency band a GW signal close to the current upper limit was predicted ${ }^{1}$ and from the PTA bounds an upper bound on the monopole-stringtension ratio was obtained, $\sqrt{\kappa} \lesssim 8[24]$.

A few months ago, the NANOGrav collaboration has reported evidence for a stochastic process at nanohertz frequencies [31], which has been interpreted as SGWB in a large number of recent papers. Possible cosmological interpretations include stable [32,33] as well as metastable strings [34]. In the latter case an analysis of the NANOGrav data deduced a monopole-string-tension ratio in the range $7.8 \leq \sqrt{\kappa} \leq 9.0$ ( $2 \sigma \mathrm{CL})$. Different string models have been compared in [35]. The possible connection of the NANOGrav results with GUT models and high-scale leptogenesis has also been studied in [36-39], and effects of low-scale leptogenesis on the SGWB were considered in [40]. Note that, independent of grand unification, a SGWB form a cosmic string network is a very interesting and well motivated signature of physics beyond the Standard Model [41-44].

The determination of the monopole-string-tension ratio $\sqrt{\kappa}$ from an interpretation of the NANOGrav data gives rise to the question whether the obtained value can be naturally realized in extensions of the Standard Model. The purpose of this paper is to clarify this question. In the following we study the simplest possible extension of the supersymmetric $\mathrm{U}(1)_{B-L}$ model considered in [17], a supersymmetric model with gauge group $\mathrm{U}(2)$, corresponding to $\mathrm{SU}(2)_{R} \times \mathrm{U}(1)_{B-L} / \mathbb{Z}_{2}$, which allows for monopoles, strings and dumbbells as topological defects.

The supersymmetric $\mathrm{U}(2)$ model has three Higgs fields, one $\mathrm{SU}(2)$ triplet and two $\mathrm{SU}(2)$ doublets. In section 2 we discuss the possible symmetry breakings and the associated defects, and in section 3 we analyze the implications for hybrid inflation. As we shall see, there are always two phases of inflation which are terminated by tachyonic preheating [45], except for one case where the symmetry is already completely broken after the first phase of inflation. The sequence of symmetry breakings is determined by the masses of the triplet and doublet Higgs fields. Depending on their ratio, either monopoles and metastable strings

\footnotetext{
${ }^{1}$ The predicted range of the dimensionless string tension is $1.0 \times 10^{-7} \leq G \mu \leq 5.6 \times 10^{-7}$, with Newton's constant $G=6.7 \times 10^{-39} \mathrm{GeV}^{-2}$. This is qualitatively consistent with the recent LIGO-Virgo upper bound on $G \mu$ for model A [30]. A quantitative comparison is not possible since the calculation in [24] uses an average power spectrum inferred from numerical simulations, assuming "cusp dominance", whereas in model A the GW spectrum is computed from the superposition of individual bursts, assuming "kink dominance".
} 
or, alternatively, dumbbells occur as topological defects. In the first case, after inflation a metastable cosmic string network is formed that leads to a SGWB in the LIGO-Virgo band, possibly extending to nanohertz frequencies. However, it is also possible that no cosmological defects survive inflation and no SGWB is generated. In the first case the reheating mechanism of the $\mathrm{U}(1)_{B-L}$ model is realized, including leptogenesis and dark matter production. In the second case, this reheating mechanism is lost. We conclude in section 4.

\section{Symmetry breaking in a supersymmetric $\mathrm{U}(2)$ model}

The starting point of cosmological $B-L$ breaking is a supersymmetric Abelian Higgs model with two chiral superfields ${ }^{2} S$ and $S_{c}$, carrying charge $q$ and $-q$, respectively, and a gauge singlet $\phi$. Kahler potential and superpotential are given by

$$
K=S^{\dagger} e^{2 g q V} S+S_{c}^{\dagger} e^{-2 g q V} S_{c}+\phi^{\dagger} \phi, \quad P=\frac{1}{4} W W+\lambda \phi\left(\frac{v_{s}^{2}}{2}-S S_{c}\right),
$$

where $V$ is a vector superfield, $W$ is the supersymmetric field strength, and $v_{s}$ is chosen real and positive. The scalar potential is given in terms of the auxiliary fields of vector and chiral superfields,

$$
\mathcal{V}=\frac{1}{2} D^{2}+\left|F_{S}\right|^{2}+\left|F_{S_{c}}\right|^{2}+\left|F_{\phi}\right|^{2}
$$

which are determined by the equations of motion,

$$
\begin{array}{ll}
D & =-g q\left(|S|^{2}-\left|S_{c}\right|^{2}\right), \\
F_{S}^{*} & =\lambda \phi S_{c}, \\
F_{S_{c}}^{*}=\lambda \phi S, \quad F_{\phi}^{*}=-\lambda\left(\frac{v_{s}^{2}}{2}-S S_{c}\right) .
\end{array}
$$

Adding to the scalar potential the kinetic terms for scalar and vector fields, one obtains for the bosonic part of the Lagrangian ${ }^{3}$

$$
\mathcal{L}_{b}=-\frac{1}{4} F_{\mu \nu} F^{\mu \nu}-\left(D_{\mu} S\right)^{*}\left(D^{\mu} S\right)-\left(D_{\mu} S_{c}\right)^{*}\left(D^{\mu} S_{c}\right)-\partial_{\mu} \phi^{*} \partial^{\mu} \phi-\mathcal{V},
$$

with the covariant derivatives $D_{\mu} S=\left(\partial_{\mu}+i g q A_{\mu}\right) S$ and $D_{\mu} S_{c}=\left(\partial_{\mu}-i g q A_{\mu}\right) S_{c}$.

The D-term leaves a flat direction, $|S|^{2}=\left|S_{c}\right|^{2}$, and the model has a continuum of supersymmetric ground states that break the U(1) symmetry,

$$
S=\frac{v_{s}}{\sqrt{2}} e^{i \alpha}, \quad S_{c}=S^{*}, \quad \phi=0 .
$$

Shifting $S$ and $S_{c}$ around their vacuum expectation values (vevs), $S=v_{s} e^{i \alpha} / \sqrt{2}+S^{\prime}$, $S_{c}=v_{s} e^{-i \alpha} / \sqrt{2}+S_{c}^{\prime}$, the Goldstone multiplet $\left(S^{\prime}-S_{c}^{\prime}\right) / \sqrt{2}$ mixes with $V$, yielding a massive vector multiplet with mass $m_{V}=\sqrt{2} g q v_{s}$, and $\left(S^{\prime}+S_{c}^{\prime}\right) / \sqrt{2}$ and $\phi$ form a massive chiral multiplet with mass $m_{S}=\lambda v_{s}$.

\footnotetext{
${ }^{2}$ We follow the conventions of ref. [46].

${ }^{3}$ We denote the vector component of $V$ by $A_{\mu}$, its field strength by $F_{\mu \nu}$, and we use the same symbol for a chiral superfield and its scalar component.
} 
The vacuum manifold $\mathcal{M}$ is a circle $S^{1}$, as in the non-supersymmetric case, with non-trivial first homotopy group, $\pi_{1}(\mathcal{M})=\mathbb{Z}$. Hence, the model has topologically stable strings as exited states. Along the D-flat direction they are given by the Nielsen-Olesen string solutions [4]. In cylindrical coordinates $(\rho, \varphi, z)$ the field configurations of static strings with winding number $n$ along the $z$ axis read

$$
S=\frac{v_{s}}{\sqrt{2}} f(\rho) e^{n i \varphi}=S_{c}^{*}, \quad A_{0}=0, \quad A_{i}=-\frac{n}{g \rho} h(\rho) \partial_{i} \varphi,
$$

where the functions $f$ and $h$ satisfy the boundary conditions $f(0)=h(0)=0, f(\infty)=$ $h(\infty)=1$. The total magnetic flux along the string is $2 n \pi / g$ and the string tension, the energy per unit length, is given by $\mu=2 \pi B(\beta) v_{s}^{2}=\pi m_{V}^{2} B(\beta) /(g q)^{2}$, where $\beta=m_{S}^{2} / m_{V}^{2}=$ $\lambda^{2} / 2 g^{2}$ and $B$ is a slowly varying function with $B(1)=1[2]$. In the following we shall consider Type-I strings with $\beta<1$, which are stable and feel an attractive force.

Cosmic strings formed in a phase transition are a source of gravitational waves. As described in the introduction, this leads to important constraints on the allowed string tension. These constraints are considerably weaker for metastable strings which naturally occur in GUT-symmetry-breaking phase transitions. Closed strings can then break into string segments connecting a monopole and an antimonopole. The simplest case where this occurs is the breaking of $\mathrm{SU}(2) \times \mathrm{U}(1)$, or more precisely $\mathrm{U}(2)=\mathrm{SU}(2) \times \mathrm{U}(1) / \mathbb{Z}_{2}$, to $\mathrm{U}(1)$. The corresponding vacuum manifold is $S^{3}$, which contains $S^{1} \cup S^{2}$, the union of the vacuum manifolds of stable strings and monopoles.

Consider first the breaking of $\mathrm{SU}(2)$ to $\mathrm{U}(1)$ by a $\mathrm{SU}(2)$ triplet $U^{a}, a=1, \ldots, 3$, which leads to topologically stable 't Hooft-Polyakov monopoles [5, 6]. Kahler potential and superpotential of the supersymmetric theory read

$$
K=U^{\dagger} e^{2 g V} U, \quad P=\frac{1}{8} \operatorname{tr}[W W],
$$

where $V=V^{a} T^{a}$ and $\left(T^{a}\right)_{b c}=-i \epsilon_{a b c}$. The bosonic part of the Lagrangian is given by

$$
\mathcal{L}_{b}=-\frac{1}{4} F_{\mu \nu}^{a} F^{a \mu \nu}-\left(D_{\mu} U^{a}\right)^{*}\left(D^{\mu} U^{a}\right)-i g \epsilon_{a b c} D^{a} U^{b *} U^{c}+\frac{1}{2} D^{a} D^{a}+F_{U}^{a *} F_{U}^{a} .
$$

where $\left(D_{\mu} U\right)^{a}=\partial_{\mu} U^{a}-g \epsilon_{a b c} A_{\mu}^{b} U^{c}$ and $F_{\mu \nu}^{a}=\partial_{\mu} A_{\nu}^{a}-\partial_{\nu} A_{\mu}^{a}-g \epsilon_{a b c} A_{\mu}^{b} A_{\nu}^{c}$. Note that this is the well known $\mathrm{SU}(2)$ Super-Yang-Mills theory with $\mathcal{N}=2$ supersymmetry $^{4}$ The classical theory has a flat direction, $U^{a}=u \delta_{a 3}$ up to gauge transformations. For this theory the full quantum and nonperturbative corrections are known. They preserve the flat direction which interpolates between a confinement phase with monopole condensation and a perturbative Higgs phase [47].

We are interested in the Higgs phase of the model, with $u$ much larger than the confinement scale $\Lambda$. As in the Abelian Higgs model, the vacuum degeneray can be lifted by adding a term to the superpotential in eq. (2.7),

$$
P=\frac{1}{8} \operatorname{tr}[W W]+\frac{\lambda^{\prime}}{2} \phi^{\prime}\left(v_{u}^{2}-U^{T} U\right)
$$

\footnotetext{
${ }^{4}$ A supersymmetric extension of the Standard Model containing a $\mathcal{N}=2$ sector with gauge group $\mathrm{SU}(2)_{R}$ could arise in an orbifold compactification of a supersymmetric Pati-Salam or $\mathrm{SO}(10)$ theory in five or six dimensions, breaking the GUT group to the Standard Model gauge group.
} 
where $\phi^{\prime}$ is a gauge singlet superfield and $v_{u}$ is chosen real and positive. The additional term in the superpotential breaks $\mathcal{N}=2$ supersymmetry to $\mathcal{N}=1$ supersymmetry. The equations of motion for the auxiliary fields are

$$
D^{a}=i g \epsilon_{a b c} U^{b *} U^{c}, \quad F_{U}^{a *}=\lambda^{\prime} \phi^{\prime} U^{a}, \quad F_{\phi^{\prime}}^{*}=-\frac{\lambda^{\prime}}{2}\left(v_{u}^{2}-U^{T} U\right) .
$$

The model has a supersymmetric vacuum,

$$
U^{a}=v_{u} \delta_{a 3}, \quad \phi^{\prime}=0,
$$

where $U^{a}$ is determined up to a $\mathrm{SU}(2)$ rotation. A $\mathrm{U}(1)$ subgroup of $\mathrm{SU}(2)$ remains unbroken. There is one massless vector multiplet, $V^{3}$, and a charged vector multiplet with mass $m_{V}=\sqrt{2} g v_{u}$, which contains the Goldstone multiplets $U^{1,2}$. The chiral multiplets $U^{\prime 3}=U^{3}-v_{u}$ and $\phi^{\prime}$ form a massive multiplet with mass $m_{U}=\lambda^{\prime} v_{u}$.

As in the non-supersymmetric case the vacuum manifold is a 2 -sphere $S^{2}$ with nontrivial homotopy group $\pi_{2}(\mathcal{M})=\mathbb{Z}$. Hence, there are topologically stable monopoles as excited states. The simplest "hedgehog" field configuration is given by $[5,6]$

$$
U^{a}=v_{u} f(r) \frac{x^{a}}{r}, \quad A_{0}^{a}=0, \quad A_{i}^{a}=h(r) \epsilon_{a i j} \frac{x^{j}}{g r^{2}},
$$

where $r=\left(x^{i} x^{i}\right)^{1 / 2}$; the functions $f$ and $h$ satisfy the boundary conditions $f(0)=h(0)=0$, $f(\infty)=h(\infty)=1$. The scalar field, and therefore the unbroken symmetry generator, points in radial direction, $\hat{\phi}^{a}=x^{a} / r$, and at large distances the gauge invariant magnetic field strength is

$$
B_{i}=-\frac{1}{2} \hat{\phi}^{a} \epsilon_{i j k} F_{j k}^{a}=\frac{x^{i}}{g r^{3}} .
$$

Hence, the magnetic charge of the monopole is $4 \pi / g$. The mass satisfies the Bogomol'nyi bound [48]

$$
m_{M} \geq \frac{4 \pi m_{V}}{g^{2}}
$$

which is saturated in the Prasad-Sommerfield limit $\lambda^{\prime} / g \rightarrow 0$ [49].

We are interested in embedding $\mathrm{U}(1)_{B-L}$ into a larger group such that $B-L$ strings can break into segments connecting a monopole with an antimonopole. The simplest possibility is to extend the electroweak part of the standard model gauge group to $\mathrm{SU}(2)_{L} \times \mathrm{SU}(2)_{R} \times$ $\mathrm{U}(1)_{B-L}$ which is then spontaneously broken to $\mathrm{SU}(2)_{L} \times \mathrm{U}(1)_{Y}$, with $Y=T_{R}^{3}+\frac{1}{2}(B-L)$. For the symmetry breaking $\mathrm{SU}(2)_{R} \times \mathrm{U}(1)_{B-L}$ to $\mathrm{U}(1)_{Y}$, the vacuum manifold is $\mathcal{M}=$ $\mathrm{U}(2) / \mathrm{U}(1)=S^{3}$. Hence, the homotopy groups $\pi_{1}(\mathcal{M})$ and $\pi_{2}(\mathcal{M})$ are trivial and there are neither topologically stable monopoles nor strings. However, as we shall see, there can be metastable strings or unstable dumbbells.

Let us now consider a supersymmetric $\mathrm{U}(2)$ model with a triplet $U$ and a pair of doublets $S, S_{c}$, transforming as $U \sim(3,0)$ and $S \sim(2, q), S_{c} \sim(\overline{2},-q)$ with respect to $\mathrm{SU}(2) \times \mathrm{U}(1)$. For previous discussions of defects in non-supersymmetric $\mathrm{U}(2)$ models with triplet and doublet scalars, see, for example, [50-53]. For the supersymmetric model, we 
choose Kahler potential and superpotential as a combination of eqs. (2.1), (2.7) and (2.9), with an additional mass term in the superpotential ${ }^{5}$

$$
\begin{aligned}
K= & U^{\dagger} e^{2 g V} U+S^{\dagger} e^{2\left(g \tilde{V}+g^{\prime} q V^{\prime}\right)} S+S_{c}^{\dagger} e^{-2\left(g \tilde{V}+g^{\prime} q V^{\prime}\right)} S_{c}+\phi^{\dagger} \phi+\phi^{\prime \dagger} \phi^{\prime}, \\
P= & \frac{1}{8} \operatorname{tr}[W W]+\frac{1}{4} W^{\prime} W^{\prime}+2 h S_{c}^{T} \tilde{U} S \\
& +\frac{\lambda^{\prime}}{2} \phi^{\prime}\left(v_{u}^{2}-U^{T} U\right)+\lambda \phi\left(\frac{v_{s}^{2}}{2}-S_{c}^{T} S\right)-h v_{u} S_{c}^{T} S,
\end{aligned}
$$

where $U=\left(U^{1}, \ldots, U^{3}\right)^{T}, V=V^{a} T^{a}, \tilde{V}=V^{a} \tau^{a} / 2$ and $\tilde{U}=U^{a} \tau^{a} / 2 ; V^{\prime}$ is the $\mathrm{U}(1)$ vector field and $W^{\prime}$ is the supersymmetric field strength. The term cubic in the Higgs fields couples the fundamental triplet $U^{a}$ to the composite triplet $S_{c}^{T} \tau^{a} S$. Without this term the group $\mathrm{U}(2)$ would in general be completely broken, and no $\mathrm{U}(1)$ subgroup would survive. The mass term $S_{c}^{T} S$ is needed in order to obtain a supersymmetric vacuum with $\langle P\rangle=0$. Supersymmetry breaking, induced by another sector, can then be hierarchically smaller than a possible $B-L$ contribution to the gravitino mass, given by $\langle P\rangle / M_{\mathrm{P}}^{2} \sim h v_{u} v_{s}^{2} / M_{\mathrm{P}}^{2}$.

The equations of motion for the auxiliary fields are

$$
\begin{aligned}
D^{a} & =-g\left(-i \epsilon_{a b c} U^{b *} U^{c}+\frac{1}{2} S^{\dagger} \tau^{a} S-\frac{1}{2} S_{c}^{\dagger} \tau^{a} S_{c}\right), \\
D & =-g^{\prime} q\left(S^{\dagger} S-S_{c}^{\dagger} S_{c}\right), \\
F_{U}^{a *} & =-h S_{c}^{T} \tau^{a} S+\lambda^{\prime} \phi^{\prime} U^{a}, \\
F_{\phi^{\prime}}^{*} & =-\frac{\lambda^{\prime}}{2}\left(v_{u}^{2}-U^{T} U\right) \\
F_{S}^{\dagger} & =-S_{c}^{T}\left(2 h \tilde{U}-\lambda \phi-h v_{u}\right), \\
F_{S_{c}}^{*} & =-\left(2 h \tilde{U}-\lambda \phi-h v_{u}\right) S, \\
F_{\phi}^{*} & =-\lambda\left(\frac{v_{s}^{2}}{2}-S_{c}^{T} S\right) .
\end{aligned}
$$

The D-terms have again flat directions and a supersymmetric vacuum exists with the expetation values

$$
U^{a}=v_{u} \delta_{a 3}, \quad S=S_{c}=\frac{v_{s}}{\sqrt{2}}\left(\begin{array}{l}
1 \\
0
\end{array}\right), \quad \phi^{\prime}=\frac{h v_{s}^{2}}{2 \lambda^{\prime} v_{u}}, \quad \phi=0 .
$$

Note that the triplets $U^{a}$ and $S_{c}^{T} \tau^{a} S$ are parallel, which is enforced by $h \neq 0$. Otherwise, the relative orientation of the two triplets would not be fixed.

In order to obtain the mass spectrum of the model, one has to shift the chiral multiplets around their vacuum expectation values: $U^{3}=v_{u}+U^{\prime 3}, S=\left(v_{s} / \sqrt{2}+S^{0^{\prime}}, S^{-}\right)^{T}, S_{c}=$ $\left(v_{s} / \sqrt{2}+S_{c}^{0^{\prime}}, S^{+}\right)^{T}, \phi^{\prime}=h v_{s}^{2} /\left(2 \lambda^{\prime} v_{u}\right)+\hat{\phi}$. From the terms of the Kahler potential (2.15) that are linear in $V, \tilde{V}$ and $V^{\prime}$, one obtains the Goldstone multiplets $\Pi^{\mp}=\left(2 v_{u} U^{\mp}+\right.$ $\left.v_{s} S^{\mp}\right) / \sqrt{4 v_{u}^{2}+v_{s}^{2}}$ and $\Pi^{0}=\left(S^{0^{\prime}}-S_{c}^{0^{\prime}}\right) / \sqrt{2}$, where $U^{ \pm}=\left(U^{1} \mp i U^{2}\right) / \sqrt{2}$. They are absorbed

\footnotetext{
${ }^{5}$ Note that this model is different from standard left-right symmetric models where triplet fields carry $\mathrm{U}(1)$ charge and therefore occur in pairs. See, for example, [54, 55].
} 
by the vector multiplets $V^{ \pm}=\left(V^{1} \mp i V^{2}\right) / \sqrt{2}$ and $V_{X}=\left(g V^{3}+2 g^{\prime} q V^{\prime}\right) /\left(2 g_{X}\right)$, respectively. The orthogonal vector multiplet $V_{Y}=\left(-2 g^{\prime} q V^{3}+g V^{\prime}\right) /\left(2 g_{X}\right)$ remains massless. The masses of the vector multiplets are

$$
m_{V}^{2}=g^{2}\left(2 v_{u}^{2}+\frac{v_{s}^{2}}{2}\right), \quad m_{X}^{2}=2 g_{X}^{2} v_{s}^{2},
$$

with

$$
g_{X}=\left(g^{\prime 2} q^{2}+\frac{g^{2}}{4}\right)^{1 / 2}, \quad \tan \Theta=\frac{2 g^{\prime} q}{g},
$$

where the angle $\Theta$ is the analogue of the weak angle in the electroweak theory.

The mass matrix of the remaining six chiral multiplets is given by the quadratic part of the superpotential,

$$
P_{m}=-h\left(\frac{4 v_{u}^{2}+v_{s}^{2}}{v_{u}}\right) \Sigma^{-} \Sigma^{+}-\lambda^{\prime} v_{u} \hat{\phi} U^{\prime 3}-v_{s}\left(\lambda \phi-h U^{\prime 3}\right) \Sigma^{0}-\frac{h v_{s}^{2}}{4 v_{u}}\left(U^{\prime 3}\right)^{2},
$$

where $\Sigma^{\mp}=\left(-v_{s} U^{\mp}+2 v_{u} S^{\mp}\right) / \sqrt{4 v_{u}^{2}+v_{s}^{2}}$ and $\Sigma^{0}=\left(S^{0^{\prime}}+S_{c}^{0^{\prime}}\right) / \sqrt{2}$ are orthogonal to the Goldstone multiples $\Pi^{\mp}$ and $\Pi^{0}$, respectively. For $h=0, P_{m}$ is the sum of the mass terms obtained above for independent $\mathrm{SU}(2)$ and $\mathrm{U}(1)$ breakings.

Depending on the parameters, the model can have two subsequent phase transitions where first $\mathrm{SU}(2)$ is broken by $\left\langle U^{a}\right\rangle$ to $\mathrm{U}(1)$, corresponding to $\mathrm{SU}(2)_{R} \rightarrow \mathrm{U}(1)_{R}$, and in a second step $\mathrm{U}(1) \times \mathrm{U}(1)$ is further broken by $\langle S\rangle$ and $\left\langle S_{c}\right\rangle$ to a $\mathrm{U}(1)$ subgroup, corresponding to $\mathrm{U}(1)_{R} \times \mathrm{U}(1)_{B-L} \rightarrow \mathrm{U}(1)_{Y}$. This is analogous to the Standard Model, where $\mathrm{SU}(2)_{L}$ contains the subgroup $\mathrm{U}(1)_{L}$ and $\mathrm{U}(1)_{L} \times \mathrm{U}(1)_{Y}$ is broken to the electromagnetic subgroup $\mathrm{U}(1)_{Q}$. The topological defects formed in the two phase transitions are monopoles and strings, respectively. Monopole mass and string tension are approximately given by

$$
m_{M} \simeq \frac{4 \pi m_{V}}{g^{2}}, \quad \mu \simeq \frac{\pi m_{X}^{2}}{g_{X}^{2}}
$$

with $m_{V}$ and $m_{X}$ given in eq. (2.18). Note that triplet and doublet vev's both contribute to the monopole mass.

In Pati-Salam- or SO(10)-GUT extensions of the Standard Model the doublets $S$ and $S_{c}$ are embedded into Pati-Salam $(4,1,2)$ - and $(\overline{4}, 1, \overline{2})$-plets, or into 16- and 16-plets of $\mathrm{SO}(10)$, respectively. ${ }^{6}$ The normalization condition $q^{2} \operatorname{tr}\left[(B-L)^{2}\right]=\operatorname{tr}\left[\left(T_{R}^{3}\right)^{2}\right]$ implies $q=\sqrt{3 / 8}$ which, together with gauge coupling unification, $g=g^{\prime}$, yields $\tan \Theta=\sqrt{3 / 2}$. For the parameter $\kappa$ that controls the metastability of cosmic strings, one obtains

$$
\kappa=\frac{m_{M}^{2}}{\mu} \simeq \frac{4 \pi}{g^{2} \cos ^{2} \Theta}\left(\frac{m_{V}}{m_{X}}\right)^{2} .
$$

As discussed in the introduction, an explanation of the NANOGrav results in terms of metastable cosmic strings requires $\sqrt{\kappa} \simeq 8$. At the GUT scale, one has $g^{2} \simeq 1 / 2$. With

\footnotetext{
${ }^{6}$ Heavy Majorana neutrino masses can be generated by the nonrenormalizable operator $1 / M_{*} h_{i j} S^{T} L_{i}^{c} S^{T} L_{j}^{c}$ where $L_{i}^{c}=\left(n_{i}^{c}, e_{i}^{c}\right)^{T}, i=1, \ldots, 3$, denote the $\mathrm{SU}(2)_{R}$ doublets of right-handed neutral and charged leptons and $h_{i j}$ are Yukawa couplings.
} 
$\cos ^{2} \Theta=2 / 5$, one obtains $\kappa \simeq 20 \pi\left(m_{V} / m_{X}\right)^{2}$. Hence, for vector-boson masses of similar size, i.e. $m_{V} \simeq m_{X}, \sqrt{\kappa} \simeq 8$ results purely from geometrical factors and the value of the gauge coupling at the GUT scale.

An important open question concerns the range of validity of the relation (2.22). For $m_{V} \simeq m_{X}$, the initial separation of the monopole-antimonopole pair is $L=2 m_{M} / \mu=$ $2 / \cos ^{2} \Theta m_{X}^{-1}=5 m_{X}^{-1}$. Hence, the thin-defect approximation made in the derivation of the decay rate (1.1), $L \gg m_{X}^{-1}$, is only marginally satisfied. Moreover, the transition from metastable strings to unstable $\mathrm{X}$-strings clearly requires a deeper analysis.

Alternatively, U(2) can be broken to U(1) by vev's of the doublets $S$ and $S_{c}$ only. The condition for this to happen will be discussed in the following section. In this case dumbbells or X-strings form which are completely analogous to the Z-strings of the Standard Model [7]. For $\tan \Theta=\sqrt{3 / 2}, \mathrm{X}$-strings are known to be unstable $[52,56]$.

\section{Hybrid inflation and the formation of monopoles, strings and dumb- bells in tachyonic preheating}

A sequence of cosmological phase transitions can lead to different types of topological defects. In our case there are two phase transitions, characterized by the vacuum expectation values $v_{s}$ and $v_{u}$. For a thermal history, the sequence of the transitions is not determined by the symmetry breaking scales but rather by the corresponding Higgs masses, i.e., $\lambda v_{s}$ and $\lambda^{\prime} v_{u}$ [53]. As we shall see, the same is true for the different phases of hybrid inflation.

Consider the evolution of the system of scalar fields along D-flat directions where the relevant part of the scalar potential is given by the F-term contributions,

$$
\begin{aligned}
\mathcal{V}_{F}= & \left(h S_{c}^{T} \tau^{a} S-\lambda^{\prime} \phi^{\prime} U^{a}\right)^{*}\left(h S_{c}^{T} \tau^{a} S-\lambda^{\prime} \phi^{\prime} U^{a}\right)+\frac{\lambda^{\prime 2}}{4}\left|v_{u}^{2}-U^{T} U\right|^{2} \\
& +S_{c}^{T}\left(2 h \tilde{U}-\lambda \phi-h v_{u}\right)\left(2 h \tilde{U}-\lambda \phi-h v_{u}\right)^{\dagger} S_{c}^{*} \\
& +S^{\dagger}\left(2 h \tilde{U}-\lambda \phi-h v_{u}\right)^{\dagger}\left(2 h \tilde{U}-\lambda \phi-h v_{u}\right) S+\frac{\lambda^{2}}{4}\left|v_{s}^{2}-2 S_{c}^{T} S\right|^{2} .
\end{aligned}
$$

The gauge-singlet fields $\phi$ and $\phi^{\prime}$ can play the role of inflatons. For $|\phi|,\left|\phi^{\prime}\right| \gg v_{s}, v_{u}$ the potential becomes

$$
\mathcal{V}_{F} \simeq \frac{\lambda^{\prime 2}}{4} v_{u}^{4}+\frac{\lambda^{2}}{4} v_{s}^{4}+\lambda^{\prime 2}\left|\phi^{\prime}\right|^{2} U^{\dagger} U+\lambda^{2}|\phi|^{2}\left(S^{\dagger} S+S_{c}^{\dagger} S_{c}\right) .
$$

The large mass terms for $U, S$ and $S_{c}$ force the waterfall fields to zero, the vacuum energy drives inflation and the potential is flat in $\phi^{\prime}$ and $\phi$. For simplicity, we choose the inflaton fields real, i.e., $\phi \equiv \varphi / \sqrt{2}=\phi^{*}$ and $\phi^{\prime} \equiv \varphi^{\prime} / \sqrt{2}=\phi^{\prime *}$. A small slope of the scalar potential is generated by the one-loop quantum corrections [10]

$$
\mathcal{V}_{1 l} \simeq \frac{3 m_{U}^{4}}{128 \pi^{2}} \ln \left(\frac{\varphi^{\prime 2}}{v_{u}^{2}}\right)+\frac{m_{S}^{4}}{32 \pi^{2}} \ln \left(\frac{\varphi^{2}}{v_{s}^{2}}\right),
$$

where $m_{U}=\lambda^{\prime} v_{u}$ and $m_{S}=\lambda v_{s}$ are the Higgs masses associated with the breaking of $\mathrm{SU}(2)$ and $\mathrm{U}(1)$, respectively, as discussed in the previous section. During the inflationary 
phase the time evolution of $\varphi$ and $\varphi^{\prime}$ is determined by the slow-roll equations of motion

$$
3 H \dot{\varphi}^{\prime}=-\partial_{\varphi^{\prime}} \mathcal{V}_{1 l}, \quad 3 H \dot{\varphi}=-\partial_{\varphi} \mathcal{V}_{1 l}
$$

where $H$ is the Hubble parameter during inflation. Starting at large field values $\varphi_{i}^{\prime}$ and $\varphi_{i}$ at an initial time $t_{i}, \varphi^{\prime}$ and $\varphi$ roll towards their critical values $v_{u}$ and $v_{s}$, respectively, where tachyonic instabilities occur. Which phase transition occurs first depends on how fast the two inflatons run, which is determined by the ratio of Higgs masses $m_{S} / m_{U}$.

\subsection{Formation of metastable strings}

Consider first the case $m_{U} \gg m_{S}$. Starting at $\varphi_{i}, \varphi_{i}^{\prime} \gg v_{s}, v_{u}$, one finds from eq. (3.4) that $\varphi^{\prime}$ reaches its critical value $v_{u}$ at the time

$$
t_{c}^{\prime}=t_{i}+\frac{32 \pi^{2} H_{i}}{m_{U}^{4}}\left(\varphi_{i}^{\prime 2}-v_{u}^{2}\right)
$$

where the Hubble parameter is $H_{i}=\sqrt{\lambda^{\prime 2} v_{u}^{4}+\lambda^{2} v_{s}^{4}} /\left(2 \sqrt{3} M_{\mathrm{P}}\right)$. At this time the inflaton field $\varphi$ takes the value

$$
\varphi\left(t_{c}^{\prime}\right)^{2}=\varphi_{i}^{2}-\frac{4}{3}\left(\frac{m_{S}}{m_{U}}\right)^{4}\left(\varphi_{i}^{\prime 2}-v_{u}^{2}\right) \simeq \varphi_{i}^{2}-\frac{4}{3}\left(\frac{m_{S}}{m_{U}}\right)^{4} \varphi_{i}^{\prime 2} .
$$

Hence, with $v_{u} \sim v_{s}$ and assuming $\varphi_{i}^{\prime} \sim \varphi_{i}$, a ratio $\lambda / \lambda^{\prime} \sim 0.1$ is already enough to ensure that at $t_{c}^{\prime}$ the inflaton field $\varphi$ is still large, $\varphi\left(t_{c}^{\prime}\right) \sim \varphi_{i}$, and therefore far away from its critical value $v_{s}$. For $\varphi^{\prime} \sim v_{u}$, the relevant part of the scalar potential reads

$$
\begin{aligned}
\mathcal{V}_{F} \simeq & \frac{\lambda^{2}}{4} v_{s}^{4}+\frac{\lambda^{2}}{2} \varphi^{2}\left(S^{\dagger} S+S_{c}^{\dagger} S_{c}\right)+\frac{m_{S}^{4}}{32 \pi^{2}} \ln \left(\frac{\varphi^{2}}{v_{s}^{2}}\right) \\
& +\frac{\lambda^{\prime 2}}{4}\left(v^{\prime 4}+2\left(\varphi^{\prime 2}-v_{u}^{2}\right) u^{T} u+2\left(\varphi^{\prime 2}+v_{u}^{2}\right) w^{T} w+\left(u^{T} u-w^{T} w\right)^{2}+4\left(u^{T} w\right)^{2}\right)
\end{aligned}
$$

where we have split $U$ into real and imaginary parts, $U^{a}=u^{a}+i w^{a}$.

The masses of $S$ and $S_{c}$ are still large and these waterfall fields therefore remain frozen at zero. Also the mass squared of $w^{a}$ is positive so that the imaginary part of $U^{a}$ stays at zero. However, the real part of $U^{a}$ becomes tachyonic, which leads to a rapid growth of the low-momentum $\left(k<k_{*}\right)$ quantum fluctuations and hence of the variance $\left\langle\left(u^{a}\right)^{2}(t)\right\rangle$. This process of "tachyonic preheating" has been investigated numerically in [45], neglecting the Hubble expansion. The root mean square value of the waterfall field can be treated as a homogeneous background field, $u^{a}(t) \simeq\left\langle\left(u^{a}\right)^{2}(t)\right\rangle^{1 / 2}$ within a patch of the size of the coherence length $\sim k_{*}^{-1}$. The growth of the fluctuations is terminated by backreaction, i.e., by the self-interaction of the waterfall field, as the different modes scatter off each other. The onset of the phase transition has also been studied analytically taking the Hubble expansion into account [57] and it has been shown that waterfall field and inflaton reach their vacuum values within a few Hubble times. The vacuum value of $u^{a}$ can be chosen as $u^{a}=v_{u} \delta_{a 3}$, and $\varphi^{\prime}=0$. During tachyonic preheating topological defects are formed, with 
a characteristic separation given by the coherence length $k_{*}^{-1}$. For the symmetry breaking $\mathrm{SU}(2)$ to $\mathrm{U}(1)$, these defects are monopoles.

After this phase of tachyonic preheating inflation continues with the inflaton $\varphi$ and the Hubble parameter $H=\lambda v_{s}^{2} /\left(2 \sqrt{3} M_{\mathrm{P}}\right)$. The critical value $\varphi_{c}=v_{s}$ is reached at time

$$
t_{c}=t_{i}+\frac{24 \pi^{2} H}{m_{S}^{4}} \varphi_{i}^{2} \gg t_{c}^{\prime}
$$

Writing $S=\left(S^{0}, S^{-}\right)^{T}$ and $S_{c}=\left(S_{c}^{0}, S^{+}\right)^{T}$, the scalar potential at $\varphi \sim v_{s}$ becomes

$$
\begin{aligned}
\mathcal{V}_{F}= & \frac{\lambda^{2}}{4} v_{s}^{4}+\frac{\lambda^{2}}{2} \varphi^{2}\left(\left|S^{0}\right|^{2}+\left|S_{c}^{0}\right|^{2}\right)+\frac{1}{2}\left(\lambda \varphi+2 \sqrt{2} h v_{u}\right)^{2}\left(\left|S^{-}\right|^{2}+\left|S^{+}\right|^{2}\right) \\
& -\lambda^{2} v_{s}^{2}\left(\operatorname{Re}\left[S_{c}^{0} S^{0}\right]+\operatorname{Re}\left[S^{+} S^{-}\right]\right)+\ldots
\end{aligned}
$$

Since SU(2) is broken the mass matrices for the $S_{c}^{0}-S^{0}$ system and the $S^{+} S^{-}$system are different. For $h v_{u} \gtrsim \lambda v_{s}$ the $S^{+}-S^{-}$mass matrix has only positive eigenvalues, and the fields $S^{+}$and $S^{-}$therefore stay at zero. In the $S_{c}^{0}-S^{0}$ system it is convenient to introduce the linear combinations $S_{1,2}=\left(\operatorname{Re}\left[S^{0} \pm S_{c}^{0}\right]+i \operatorname{Im}\left[S^{0} \mp S_{c}^{0}\right]\right) / \sqrt{2}$. The scalar potential for the fields $S_{1,2}, \varphi$ and $\varphi^{\prime}$ reads

$$
\begin{aligned}
\mathcal{V}_{F}= & \frac{1}{4}\left(h\left(\left|S_{1}\right|^{2}-\left|S_{2}\right|^{2}\right)-\sqrt{2} \lambda^{\prime} \varphi^{\prime} v_{u}\right)^{2}+\frac{h^{2}+\lambda^{2}}{4}\left(\operatorname{Im}\left[S_{1} S_{2}\right]\right)^{2} \\
& +\frac{\lambda^{2}}{4}\left(v_{s}^{4}+2\left(\varphi^{2}-v_{s}^{2}\right)\left|S_{1}\right|^{2}+2\left(\varphi^{2}+v_{s}^{2}\right)\left|S_{2}\right|^{2}+\left(\left|S_{1}\right|^{2}-\left|S_{2}\right|^{2}\right)^{2}\right) .
\end{aligned}
$$

At $\varphi=v_{s}$, the waterfall field $S_{1}$ becomes tachyonic whereas the mass squared of $S_{2}$ remains positive. This leads to a second phase of tachyonic preheating where a $U(1)$ symmetry is broken and cosmic strings are formed. Within a few Hubble times inflaton and waterfall field reach their vacuum values $S_{1}=v_{s}$ and $\varphi=0$. The vev of $S_{1}$ generates a tadpole for $\varphi^{\prime}$, which is therefore shifted from $\varphi^{\prime}=0$ to $\varphi^{\prime}=h v_{s}^{2} /\left(\sqrt{2} \lambda^{\prime} v_{u}\right)$.

The total number of $e$-folds generated in the second phase of inflation is

$$
N\left(\varphi\left(t_{c}^{\prime}\right)\right)=\int_{t\left(\varphi_{c}\right)}^{t_{c}^{\prime}} H d t=\frac{4 \pi^{2}}{\lambda^{2} M_{\mathrm{P}}{ }^{2}}\left(\varphi\left(t_{c}^{\prime}\right)^{2}-v_{s}^{2}\right) \simeq \frac{4 \pi^{2}}{\lambda^{2} M_{\mathrm{P}}{ }^{2}} \varphi_{i}^{2}
$$

where we have used that inflation ends at the critical field value $\varphi_{c}=v_{s}$, and the second equation holds for the considered parameters. The field value corresponding to the last 50 $e$-folds is denoted as $\varphi_{*}$, i.e., $N\left(\varphi_{*}\right) \equiv N_{*}=50$. The reheating process after inflation has been studied in detail in $[17,19]$. Successful leptogenesis restricts the reheating temperature to $10^{8} \mathrm{GeV} \lesssim T_{\mathrm{rh}} \lesssim 10^{10} \mathrm{GeV}$. Since the reheating process is triggered by Higgs decays, this constrains the Higgs mass, and therefore the Higgs coupling to $\lambda \sim 10^{-3}$ within about one order of magnitude. For Higgs couplings in this range, the inflationary observables, given in terms of the slow-roll parameters

$$
\epsilon=\frac{M_{\mathrm{P}}^{2}}{2}\left(\frac{\partial_{\varphi} V}{V}\right)^{2}, \quad \eta=M_{\mathrm{P}}^{2} \frac{\partial_{\varphi}^{2} V}{V},
$$


satifsfy the simple relations,

$$
\begin{aligned}
& A_{s}=\left.\frac{H^{2}}{8 \pi^{2} \epsilon M_{\mathrm{P}}^{2}}\right|_{\varphi_{*}} \simeq \frac{1}{3}\left(\frac{v_{s}}{M_{\mathrm{P}}}\right)^{4} N_{*}, \\
& n_{s} \simeq 1+\left.2 \eta\right|_{\varphi_{*}} \simeq 1-\frac{1}{N_{*}} \simeq 0.98,
\end{aligned}
$$

where $A_{s}$ and $n_{s}$ are the amplitude of scalar fluctuations and the scalar spectral index, respectively. With $A_{s}=(2.099 \pm 0.029) \times 10^{-9}$ [58], one obtains from eq. (3.13) a symmetry breaking vev of order the GUT scale, $v \approx 5 \times 10^{15} \mathrm{GeV}$. The tensor-to-scalar ratio turns out to be very small, $r=A_{t} / A_{s}=\left.16 \epsilon\right|_{\varphi_{*}} \simeq \lambda^{2} /\left(2 \pi^{2} N_{*}\right) \lesssim 10^{-7}$. The predicted scalar spectral index $n_{s}$ is consistent at $3 \sigma$ CL with the observed value $n_{s}=0.9649 \pm 0.0042$ [58]. The agreement with observation can be improved by taking the supergravity effect of a constant $P_{0}$ in the superpotential into account, which is related to the gravitino mass as $P_{0}=\alpha m_{3 / 2} M_{\mathrm{P}}$, where $\alpha \sim 1$ encodes the details of supersymmetry breaking. Remarkably, the predicted scalar spectral index turns out to be consistent with observation for gravitino masses in the range $10 \mathrm{TeV} \lesssim m_{3 / 2} \lesssim 10 \mathrm{PeV}$. Variation of the gravitino mass in this range is connected to a variation of the Higgs coupling $\lambda$ and the reheating temperature by about two orders of magnitude. A detailed discussion can be found in $[18,24]$.

For generic initial values $\varphi_{i} \gg v_{s}$, the total number of $e$-folds during the second phase of inflation is much larger than 50, and the original density of monopoles is completely diluted. The strings produced in the second phase of tachyonic preheating are metastable, i.e., long string loops tunnel to configurations of equal energy, consisting of string segments that connect monopole-antimonopole pairs [26]. The geometry of these configurations is reviewed in [53]. Consider the monopole at one end of a string segment. At large distances away from the center of the monopole the triplet field has the familiar hedgehog structure (2.12), so that in polar coordinates $(r, \alpha, \beta)$,

$$
\tilde{U} \propto \frac{x^{a}}{r} \tau^{a}=\left(\begin{array}{cc}
\cos \alpha & \sin \alpha e^{-i \beta} \\
\sin \alpha e^{i \beta} & -\cos \alpha
\end{array}\right) .
$$

To minimize the energy density of the configuration, $U, S$ and $S_{c}$ have to be "aligned" around the sphere. From the scalar potential (3.1) one reads off that $S$ and $S_{c}$ have to be eigenvectors of $\tilde{U}$ with postive eigenvalue in all directions, which corresponds to [7]

$$
S_{c}=S \propto\left(\begin{array}{c}
\cos \frac{\alpha}{2} \\
\sin \frac{\alpha}{2} e^{i \beta}
\end{array}\right)
$$

Because of the phase factor, these eigenvectors are singular at the south pole $\alpha=\pi$. Here a string must be attached, around which the phases of $S$ and $S_{c}$ change by $2 \pi$.

\subsection{Formation of dumbbells}

Consider now the opposite case $m_{S} \gg m_{U}$. Now $\varphi$ reaches its critical value $\varphi_{c} \sim v_{s}$ first, at a time

$$
t_{c}=t_{i}+\frac{24 \pi^{2} H_{i}}{m_{S}^{4}} \varphi_{i}^{2}
$$


when $\varphi^{\prime}$ is still much larger than $v_{u}$. For $\varphi \sim \varphi_{c}$, the scalar potential reads

$$
\begin{aligned}
\mathcal{V}_{F} \simeq & \frac{\lambda^{\prime 2}}{4} v_{u}^{4}+\frac{\lambda^{\prime 2}}{2} \varphi^{\prime 2} U^{\dagger} U+\frac{3 m_{U}^{4}}{128 \pi^{2}} \ln \left(\frac{\varphi^{\prime 2}}{v_{u}^{2}}\right) \\
& +\frac{\lambda^{2}}{4}\left(v_{s}^{4}+2 \hat{\varphi}^{2}\left(S^{\dagger} S+S_{c}^{\dagger} S_{c}\right)-4 v_{s}^{2} \operatorname{Re}\left[S_{c}^{T} S\right]+4\left|S_{c}^{T} S\right|^{2}\right)+\ldots,
\end{aligned}
$$

where $\hat{\varphi}=\varphi+\sqrt{2} h v_{u} / \lambda$. The potential is invariant under $\mathrm{SU}(2) \times \mathrm{U}(1)$. At a critical value $\varphi_{c}$, the waterfall fields $S$ and $S_{c}$ become tachyonic along the D-flat directions. Their vev's can be chosen to be the upper components of $S$ and $S_{c}$. Switching again from $S^{0}$ and $S_{c}^{0}$ to $S_{1,2}$, the scalar potential becomes

$$
\begin{aligned}
\mathcal{V}_{F} \simeq & \frac{\lambda^{\prime 2}}{4} v_{u}{ }^{4}+\frac{\lambda^{\prime 2}}{2} \varphi^{\prime 2} U^{\dagger} U+\frac{3 m_{U}^{4}}{128 \pi^{2}} \ln \left(\frac{\varphi^{\prime 2}}{v_{u}^{2}}\right)+\frac{\lambda^{2}}{4}\left(v_{s}^{4}+2\left(\hat{\varphi}^{2}-v_{s}^{2}\right)\left|S_{1}\right|^{2}\right. \\
& \left.+2\left(\hat{\varphi}^{2}+v_{s}^{2}\right)\left|S_{2}\right|^{2}+\left(\left|S_{1}\right|^{2}-\left|S_{2}\right|^{2}\right)^{2}+\left(\operatorname{Im}\left[S_{1} S_{2}\right]\right)^{2}\right)+\ldots
\end{aligned}
$$

At the critical value $\varphi_{c}=v_{s}-\sqrt{2} h v_{u} / \lambda$, the field $S_{1}$ becomes tachyonic and in a few Hubble times the system reaches the vacuum configuration $S_{1}=v_{s}, \varphi=-\sqrt{2} h v_{u} / \lambda$. The field $S_{2}$ stays at zero.

The symmetry is now broken from $\mathrm{SU}(2) \times \mathrm{U}(1) / \mathbb{Z}_{2}$ to $\mathrm{U}(1)$ and the vacuum manifold is $\mathrm{U}(2) / \mathrm{U}(1)=S^{3}$. As discussed in the previous section, there are no topologically stable strings, but instead dumbbells or X-strings, analogous to the Z-strings of the Standard Model. These defects are unstable. ${ }^{7}$

After tachyonic preheating inflation continues with $\varphi^{\prime}$ as inflaton and the critical value $\varphi_{c}^{\prime}$ is reached at time

$$
t_{c}^{\prime} \simeq t_{i}+\frac{32 \pi^{2} H^{\prime}}{m_{U}^{4}} \varphi_{i}^{\prime 2}
$$

where now the Hubble parameter is $H^{\prime}=\lambda^{\prime} v_{u}^{2} /\left(2 \sqrt{3} M_{\mathrm{P}}\right)$.

With $S$ and $S_{c}$ settled at their vacuum values, and splitting again the waterfall fields $U^{a}$ into real and imaginary parts, $U^{a}=u^{a}+i w^{a}$, the scalar potential for $u^{a}$ and $\varphi^{\prime}$ reads

$$
\mathcal{V}_{F}=\frac{h^{2}}{4} v_{s}^{4}+\frac{3 m_{U}^{4}}{128 \pi^{2}} \ln \left(\frac{\varphi^{\prime 2}}{v_{u}^{2}}\right)-\frac{h \lambda^{\prime}}{\sqrt{2}} v_{s}^{2} \varphi^{\prime} u^{3}+\frac{\lambda^{\prime 2}}{2} \varphi^{\prime 2} u^{a} u^{a}+\ldots
$$

Contrary to the potentials (3.7), (3.10) and (3.18), now a term linear in the waterfall field $U$ appears. This reflects the fact that after the condensation of $S$ and $S_{c}$ the symmetry $\mathrm{U}(2)$ is already broken to the final $\mathrm{U}(1)$ subgroup, and hence also $u^{3}$ gets a small vev which for large values of $\varphi^{\prime}$ reads

$$
u^{3} \simeq \frac{\sqrt{2}}{h \lambda^{\prime} v_{s}^{2}} \frac{1}{\varphi^{\prime}}\left(\frac{h^{2}}{4} v_{s}^{4}+\frac{3 m_{U}^{4}}{128 \pi^{2}} \ln \left(\frac{\varphi^{\prime 2}}{v_{u}^{2}}\right)\right)+\ldots
$$

\footnotetext{
${ }^{7}$ For a numerical simulation of dumbbell production during a phase transition, see [50].
} 
As $\varphi^{\prime}$ decreases, $u^{3}$ increases until eventually all fields are of the order of $v_{u}, v_{s}$. The scalar potential for the fields $u^{a}, w^{a}, \varphi$ and $\varphi^{\prime}$ is given by $(i=1,2, a=1, \ldots, 3)$

$$
\begin{aligned}
\mathcal{V}_{F}= & \left(\frac{h}{2} v_{s}^{2}-\frac{\lambda^{\prime}}{\sqrt{2}} \varphi^{\prime} u^{3}\right)^{2}+\frac{\lambda^{\prime 2}}{4}\left(\left(v_{u}^{2}-u^{a} u^{a}+w^{a} w^{a}\right)^{2}+4\left(u^{a} w^{a}\right)^{2}\right) \\
& +\frac{\lambda^{\prime 2}}{2} \varphi^{\prime 2}\left(u^{i} u^{i}+w^{a} w^{a}\right)+v_{s}^{2}\left(\left(h u^{3}-\frac{\lambda}{\sqrt{2}} \varphi-h v_{u}\right)^{2}+h^{2}\left(u^{i} u^{i}+w^{a} w^{a}\right)\right) .
\end{aligned}
$$

Backreaction between waterfall fields and inflaton fields leads to oscillations, and eventually the fields settle in the supersymmetric vacuum $u^{3}=v_{u}, u^{i}=w^{a}=0, \varphi=0$ and $\varphi^{\prime}=$ $h v_{s}^{2} /\left(\sqrt{2} v_{u}\right)$. Hence, there is no second phase of tachyonic preheating and there are no topological defects that survive inflation. Therefore, there is no SGWB produced by a string network and also the reheating mechanism described in the previous section is lost.

\section{Conclusions}

We have studied symmetry breaking and topological defects in a supersymmetric model with gauge group $\mathrm{U}(2)=\mathrm{SU}(2)_{R} \times \mathrm{U}(1)_{B-L} / \mathbb{Z}_{2}$, which is the simplest non-Abelian extension of a previously considered model with local $\mathrm{U}(1)_{B-L}$ symmetry. The model has a triplet $U$ and two doublets, $S$ and $S_{c}$ of chiral superfields. To lift the vacuum degeneracy of the D-term potential two gauge singlet chiral superfields are needed, which can naturally play the role of inflatons.

The model has various topological defects. The breaking of $\mathrm{SU}(2)$ to $\mathrm{U}(1)$ by a vev of $U$ leads to monopoles, and the subsequent breaking of $U(1) \times U(1)$ to $U(1)$ by vev's of $S$ and $S_{c}$ leads to metastable strings. They are classically stable but decay by quantum tunneling. The breaking of U(2) to U(1) by vev's of $S$ and $S_{c}$ only leads to unstable dumbbells or X-strings, analogous to Z-strings in the Standard Model.

Which sequence of symmetry breakings is realized depends for thermal phase transitions on the ratio of Higgs masses $m_{S}^{2} / m_{U}^{2}$. The same is true for hybrid inflation. Starting at large initial values of the two inflaton fields, the speed of their slow-roll motion is proportional to $m_{S}^{4}$ and $m_{U}^{4}$, respectively. For $m_{U} \gg m_{S}$, first $U$ condenses and monopoles are formed in tachyonic preheating. In the subsequent phase of inflation the monopoles are diluted and in the second phase of tachyonic preheating metastable strings are produced. Alternatively, for $m_{S}^{2} \gg m_{U}^{2}$, first $S$ and $S_{c}$ condense and unstable dumbbells are formed. In the second phase of inflation, the expectation value of $U$ smoothly evolves from zero to its vacuum value and there is no second phase of tachyonic preheating.

Only in the case $m_{U} \gg m_{S}$ a metastable string network is formed which can generate a stochastic gravitational wave background. If the reheating process is required to include thermal or non-thermal leptogenesis, the reheating temperature has to be larger than about $10^{8} \mathrm{GeV}$. This implies a lower bound on the Higgs mass $m_{S}$ and the Higgs coupling $\lambda$, such that the string tension is predicted in a narrow range, $G \mu=(1.0-5.6) \times 10^{-7}$. This leads to the prediction of a SGWB with amplitude $h^{2} \Omega_{\mathrm{GW}} \sim 10^{-8}$ in the LIGO-Virgo frequency band. The spectrum of the SGWB depends exponentially on the monopolestring-tension ratio $\sqrt{\kappa}$. For $\sqrt{\kappa} \simeq 8$ the evidence for a stochastic process recently reported 
by the NANOGrav collaboration can be interpreted as SGWB from a metastable cosmic string network. However, already for $\sqrt{\kappa} \simeq 6$ the SGWB amplitude is cut off above the LIGO-Virgo frequency band. In the considered model, for approximately equal symmetry breaking scales, the value $\sqrt{\kappa} \simeq 8$ is obtained from geometrical factors and the value of the gauge coupling at the GUT scale.

\section{Acknowledgments}

I thank Valerie Domcke and Kai Schmitz for valuable discussions and comments on the manuscript.

Open Access. This article is distributed under the terms of the Creative Commons Attribution License (CC-BY 4.0), which permits any use, distribution and reproduction in any medium, provided the original author(s) and source are credited.

\section{References}

[1] T.W.B. Kibble, Topology of cosmic domains and strings, J. Phys. A 9 (1976) 1387 [InSPIRE].

[2] M. Hindmarsh, Signals of inflationary models with cosmic strings, Prog. Theor. Phys. Suppl. 190 (2011) 197 [arXiv:1106.0391] [INSPIRE].

[3] P. Auclair et al., Probing the gravitational wave background from cosmic strings with LISA, JCAP 04 (2020) 034 [arXiv: 1909.00819] [InSPIRE].

[4] H.B. Nielsen and P. Olesen, Vortex line models for dual strings, Nucl. Phys. B 61 (1973) 45 [INSPIRE].

[5] G. 't Hooft, Magnetic monopoles in unified gauge theories, Nucl. Phys. B 79 (1974) 276 [INSPIRE].

[6] A.M. Polyakov, Particle spectrum in the quantum field theory, JETP Lett. 20 (1974) 194 [Pisma Zh. Eksp. Teor. Fiz. 20 (1974) 430] [inSPIRE].

[7] Y. Nambu, String-like configurations in the Weinberg-Salam theory, Nucl. Phys. B 130 (1977) 505 [INSPIRE].

[8] T.W.B. Kibble, G. Lazarides and Q. Shafi, Strings in SO(10), Phys. Lett. B 113 (1982) 237 [INSPIRE].

[9] E.J. Copeland, A.R. Liddle, D.H. Lyth, E.D. Stewart and D. Wands, False vacuum inflation with Einstein gravity, Phys. Rev. D 49 (1994) 6410 [astro-ph/9401011] [INSPIRE].

[10] G.R. Dvali, Q. Shafi and R.K. Schaefer, Large scale structure and supersymmetric inflation without fine tuning, Phys. Rev. Lett. 73 (1994) 1886 [hep-ph/9406319] [INSPIRE].

[11] R. Jeannerot, J. Rocher and M. Sakellariadou, How generic is cosmic string formation in SUSY GUTs, Phys. Rev. D 68 (2003) 103514 [hep-ph/0308134] [INSPIRE].

[12] P. Minkowski, $\mu \rightarrow$ ey at a rate of one out of $10^{9}$ muon decays?, Phys. Lett. B 67 (1977) 421 [INSPIRE].

[13] T. Yanagida, Horizontal gauge symmetry and masses of neutrinos, Conf. Proc. C $\mathbf{7 9 0 2 1 3 1}$ (1979) 95 [INSPIRE]. 
[14] M. Gell-Mann, P. Ramond and R. Slansky, Complex spinors and unified theories, Conf. Proc. C 790927 (1979) 315 [arXiv: 1306.4669] [INSPIRE].

[15] M. Fukugita and T. Yanagida, Baryogenesis without grand unification, Phys. Lett. B 174 (1986) 45 [INSPIRE].

[16] D. Bödeker and W. Buchmüller, Baryogenesis from the weak scale to the grand unification scale, arXiv:2009.07294 [INSPIRE].

[17] W. Buchmüller, V. Domcke and K. Schmitz, Spontaneous B-L breaking as the origin of the hot early universe, Nucl. Phys. B 862 (2012) 587 [arXiv:1202.6679] [INSPIRE].

[18] W. Buchmüller, V. Domcke, K. Kamada and K. Schmitz, Hybrid inflation in the complex plane, JCAP 07 (2014) 054 [arXiv: 1404.1832] [INSPIRE].

[19] W. Buchmüller, V. Domcke, K. Kamada and K. Schmitz, The gravitational wave spectrum from cosmological B-L breaking, JCAP 10 (2013) 003 [arXiv: 1305.3392] [INSPIRE].

[20] J.A. Dror, T. Hiramatsu, K. Kohri, H. Murayama and G. White, Testing the seesaw mechanism and leptogenesis with gravitational waves, Phys. Rev. Lett. 124 (2020) 041804 [arXiv: 1908.03227] [INSPIRE].

[21] NANOGRAV collaboration, The NANOGrav 11-year data set: pulsar-timing constraints on the stochastic gravitational-wave background, Astrophys. J. 859 (2018) 47 [arXiv: 1801.02617] [INSPIRE].

[22] M. Kerr et al., The Parkes pulsar timing array project: second data release, Publ. Astron. Soc. Austral. 37 (2020) e020 [arXiv: 2003.09780] [InSPIRE].

[23] R.M. Shannon et al., Gravitational waves from binary supermassive black holes missing in pulsar observations, Science 349 (2015) 1522 [arXiv:1509.07320] [INSPIRE].

[24] W. Buchmüller, V. Domcke, H. Murayama and K. Schmitz, Probing the scale of grand unification with gravitational waves, Phys. Lett. B 809 (2020) 135764 [arXiv:1912.03695] [INSPIRE].

[25] A. Vilenkin, Cosmological evolution of monopoles connected by strings, Nucl. Phys. B 196 (1982) 240 [INSPIRE].

[26] J. Preskill and A. Vilenkin, Decay of metastable topological defects, Phys. Rev. D 47 (1993) 2324 [hep-ph/9209210] [INSPIRE].

[27] L. Leblond, B. Shlaer and X. Siemens, Gravitational waves from broken cosmic strings: the bursts and the beads, Phys. Rev. D 79 (2009) 123519 [arXiv:0903.4686] [INSPIRE].

[28] A. Monin and M.B. Voloshin, The spontaneous breaking of a metastable string, Phys. Rev. D 78 (2008) 065048 [arXiv: 0808.1693] [INSPIRE].

[29] J.J. Blanco-Pillado, K.D. Olum and B. Shlaer, The number of cosmic string loops, Phys. Rev. D 89 (2014) 023512 [arXiv:1309.6637] [InSPIRE].

[30] LIGO Scientific, Virgo and KAGRA collaborations, Constraints on cosmic strings using data from the third advanced LIGO-Virgo observing run, arXiv:2101.12248 [INSPIRE].

[31] NANOGRAV collaboration, The NANOGrav 12.5 yr data set: search for an isotropic stochastic gravitational-wave background, Astrophys. J. Lett. 905 (2020) L34 [arXiv: 2009. 04496] [INSPIRE].

[32] J. Ellis and M. Lewicki, Cosmic string interpretation of NANOGrav pulsar timing data, Phys. Rev. Lett. 126 (2021) 041304 [arXiv:2009.06555] [INSPIRE]. 
[33] S. Blasi, V. Brdar and K. Schmitz, Has NANOGrav found first evidence for cosmic strings?, Phys. Rev. Lett. 126 (2021) 041305 [arXiv: 2009. 06607] [INSPIRE].

[34] W. Buchmüller, V. Domcke and K. Schmitz, From NANOGrav to LIGO with metastable cosmic strings, Phys. Lett. B 811 (2020) 135914 [arXiv: 2009.10649] [INSPIRE].

[35] J.J. Blanco-Pillado, K.D. Olum and J.M. Wachter, Comparison of cosmic string and superstring models to NANOGrav 12.5 year results, arXiv:2102.08194 [INSPIRE].

[36] S. Chigusa, Y. Nakai and J. Zheng, Implications of gravitational waves for supersymmetric grand unification, arXiv:2011.04090 [INSPIRE].

[37] J. Chakrabortty, G. Lazarides, R. Maji and Q. Shafi, Primordial monopoles and strings, inflation, and gravity waves, JHEP 02 (2021) 114 [arXiv: 2011.01838] [INSPIRE].

[38] R. Samanta and S. Datta, Gravitational wave complementarity and impact of NANOGrav data on gravitational leptogenesis: cosmic strings, arXiv:2009.13452 [INSPIRE].

[39] S.F. King, S. Pascoli, J. Turner and Y.-L. Zhou, Gravitational waves and proton decay: complementary windows into grand unified theories, Phys. Rev. Lett. 126 (2021) 021802 [arXiv: 2005.13549] [INSPIRE].

[40] S. Blasi, V. Brdar and K. Schmitz, Fingerprint of low-scale leptogenesis in the primordial gravitational-wave spectrum, Phys. Rev. Res. 2 (2020) 043321 [arXiv:2004.02889] [INSPIRE].

[41] Y. Cui, M. Lewicki, D.E. Morrissey and J.D. Wells, Cosmic archaeology with gravitational waves from cosmic strings, Phys. Rev. D 97 (2018) 123505 [arXiv:1711.03104] [InSPIRE].

[42] Y. Cui, M. Lewicki, D.E. Morrissey and J.D. Wells, Probing the pre-BBN universe with gravitational waves from cosmic strings, JHEP 01 (2019) 081 [arXiv:1808.08968] [INSPIRE].

[43] Y. Gouttenoire, G. Servant and P. Simakachorn, Beyond the Standard Models with cosmic strings, JCAP 07 (2020) 032 [arXiv:1912.02569] [INSPIRE].

[44] Y. Gouttenoire, G. Servant and P. Simakachorn, BSM with cosmic strings: heavy, up to EeV mass, unstable particles, JCAP 07 (2020) 016 [arXiv: 1912.03245] [INSPIRE].

[45] G.N. Felder, J. García-Bellido, P.B. Greene, L. Kofman, A.D. Linde and I. Tkachev, Dynamics of symmetry breaking and tachyonic preheating, Phys. Rev. Lett. 87 (2001) 011601 [hep-ph/0012142] [INSPIRE].

[46] J. Wess and J. Bagger, Supersymmetry and supergravity, Princeton University Press, Princeton, NJ, U.S.A. (1992).

[47] N. Seiberg and E. Witten, Electric-magnetic duality, monopole condensation, and confinement in $N=2$ supersymmetric Yang-Mills theory, Nucl. Phys. B 426 (1994) 19 [Erratum ibid. 430 (1994) 485] [hep-th/9407087] [INSPIRE].

[48] E.B. Bogomolny, Stability of classical solutions, Sov. J. Nucl. Phys. 24 (1976) 449 [Yad. Fiz. 24 (1976) 861] [INSPIRE].

[49] M.K. Prasad and C.M. Sommerfield, An exact classical solution for the 't Hooft monopole and the Julia-Zee dyon, Phys. Rev. Lett. 35 (1975) 760 [InSPIRE].

[50] E.J. Copeland, D. Haws, T.W.B. Kibble, D. Mitchell and N. Turok, Monopoles connected by strings and the monopole problem, Nucl. Phys. B 298 (1988) 445 [INSPIRE].

[51] T.W. Kephart and T. Vachaspati, Topological incarnations of electroweak defects, Phys. Lett. B 388 (1996) 481 [hep-ph/9503355] [INSPIRE]. 
[52] A. Achucarro and T. Vachaspati, Semilocal and electroweak strings, Phys. Rept. 327 (2000) 347 [hep-ph/9904229] [INSPIRE].

[53] T.W.B. Kibble and T. Vachaspati, Monopoles on strings, J. Phys. G 42 (2015) 094002 [arXiv: 1506.02022] [INSPIRE].

[54] C.S. Aulakh, A. Melfo and G. Senjanović, Minimal supersymmetric left-right model, Phys. Rev. D 57 (1998) 4174 [hep-ph/9707256] [INSPIRE].

[55] K.S. Babu and R.N. Mohapatra, Minimal supersymmetric left-right model, Phys. Lett. B 668 (2008) 404 [arXiv: 0807.0481] [INSPIRE].

[56] T. Vachaspati, Vortex solutions in the Weinberg-Salam model, Phys. Rev. Lett. 68 (1992) 1977 [Erratum ibid. 69 (1992) 216] [INSPIRE].

[57] T. Asaka, W. Buchmüller and L. Covi, False vacuum decay after inflation, Phys. Lett. B 510 (2001) 271 [hep-ph/0104037] [INSPIRE].

[58] Planck collaboration, Planck 2018 results. X. Constraints on inflation, Astron. Astrophys. 641 (2020) A10 [arXiv: 1807.06211] [INSPIRE]. 